\title{
Increase in Plasma Lipid Peroxide in Cats Fed a Fish Diet
}

\author{
Yasuyuki MOMOI ${ }^{1)}$, Yuko GOTO ${ }^{2)}$, Kyoko TANIDE ${ }^{1)}$, Noriko TAKAHASHI ${ }^{2)}$, Toshihiro WATARI ${ }^{3)}$, \\ Kazuaki YAMAZOE ${ }^{1)}$, Hajime TSUJIMOTO ${ }^{2)}$ and Tadaaki KUDO ${ }^{1)}$ \\ ${ }^{1)}$ Department of Veterinary Surgery, Faculty of Agriculture, Gifu University, 1-1 Yanagido, Gifu 501-1112, ${ }^{2)}$ Department of Veterinary \\ Internal Medicine, Graduate School of Agriculture and Life Sciences, The University of Tokyo, 1-1-1 Yayoi, Bunkyo-ku, Tokyo 113-8657, \\ and ${ }^{3)}$ Laboratory of Comprehensive Veterinary Clinical Studies, College of Bioresource Sciences, NIHON University, 1866 Kameino, \\ Fujisawa, Kanagawa 252-8510, Japan
}

(Received 5 September 2000/Accepted 21 August 2001)

ABSTRACT. Plasma lipid peroxide levels were examined in cats. Plasma lipid peroxide levels in 3 of 4 clinical cases which had been fed raw fish were higher than those in normal cats. When healthy cats were put on a raw fish diet in controlled conditions, a remarkable increase in plasma lipid peroxide was observed. This increase occurred within 1 to 3 weeks in cats without obvious clinical disorders. We also showed that a continuous raw fish diet is necessary to cause the increase, but the increase was transient and restored in spite of a continuous raw fish diet, indicating the development of an unidentified antioxidant process. Our results clearly indicate that intake of foods high in polyunsaturated fatty acid can induce oxidative stress in cats.

KEY WORDS: feline, fish diet, lipid peroxide, polyunsaturated fatty acid.

J. Vet. Med.Sci. 63(12): 1293-1296, 2001

Nowadays the medical importance of free radicals is emphasized. When any free radical species is generated in vivo, lipid peroxides and related radicals are produced from lipid component polyunsaturated fatty acid $[15,18,19]$. They can denature proteins, enzymes, lipids and nucleic acids, resulting in injury to cells, organs and tissues $[15,18$, 19]. When lipid peroxides form and accumulate to a certain degree, they leak from the organ or tissue into the bloodstream and increase the lipid peroxide level in the serum and plasma. Although an increase in lipid peroxide in the blood can be due to either food intake or endogenous causes, it has been believed that an abnormal increase in the blood lipid peroxide level is usually due to endogenous causes [18]. Clinically, because of their short half-life, measuring lipid free radicals is difficult, and therefore relatively stable substances such as lipid hydroperoxides and aldehydes with a long carbon chain were measured by the thiobarbituric acid (TBA) method as lipid peroxide [17] and the methylcarbamoyl-dimethylamino phenothizine-Hb (MCDP-Hb) method is also applied to measure lipid hydroperoxide as lipid peroxide [10]. Current evidence has indicated that there is a correlation between serum lipid peroxide levels and some generalized disorders in humans, including dermatoses, atheroscleroses and diabetes mellitus $[2,3,4,6,7,11,12,14$, 16]. But the effect of diet on an increase in the lipid peroxide level in blood has been studied insufficiently. Lipid peroxides and related radicals must be associated with the pathogenesis of some animal diseases such as pansteatis in cats as well as human diseases, but the medical importance of lipid peroxides and related radicals has not been investigated in veterinary medicine. Furthermore, the blood lipid peroxide level has not been measured and its clinical significance has not been evaluated.

Domestic cats are often fed either raw fish or commercial cat food containing fish. The fish oil usually contains large amounts of highly unsaturated fatty acids. In this study, we measured plasma lipid peroxide levels in several cat diseases and investigated the effect of a fish diet on changes in plasma lipid peroxide levels in cats for several weeks.

\section{MATERIALS AND METHODS}

Subjects: In the control group, plasma lipid peroxide levels were determined in 15 clinical healthy cats. The test group consisted of five household cats who were fed raw fish as their principal food and three cats with insulin dependent diabetes mellitus (IDDM) from the Veterinary Medical Center of the University of Tokyo. We also used five clinical healthy cats which had been fed a commercial cat food made of beef then fed a raw fish (mackerel or horse mackerel) diet for 1 to 6 weeks. Approximately, $60 \mathrm{~g} / \mathrm{kg} /$ day of raw fish was fed by ad lib. feeding or compulsively. We also collected blood samples from these cats and reacted them with air by gentle continuous stirring for $5 \mathrm{hr}$ at $38^{\circ} \mathrm{C}$ to evaluate the potential plasma lipid peroxide.

Assay for plasma lipid peroxide: Heparinized blood plasma was collected and the plasma lipid peroxide level was evaluated by the thiobarbituric acid (TBA) method [17] or methyl carbamoyl-dimethylamino phenothiazine-Hb (MCDP) method [10].

\section{RESULTS}

Plasma lipid peroxide levels in clinical cases: The levels of plasma lipid peroxide in clinically healthy cats were determined by the TBA method (Fig. 1). The mean value was $1.5 \mathrm{nmol} / \mathrm{m} l$ and the highest did not exceed $3.0 \mathrm{nmol} /$ $\mathrm{m} l$. In the three cats with IDDM and the five cats fed raw fish, one of three cats with IDDM had a high plasma lipid peroxide level $(3.4 \mathrm{nmol} / \mathrm{ml})$, though it was close to the 


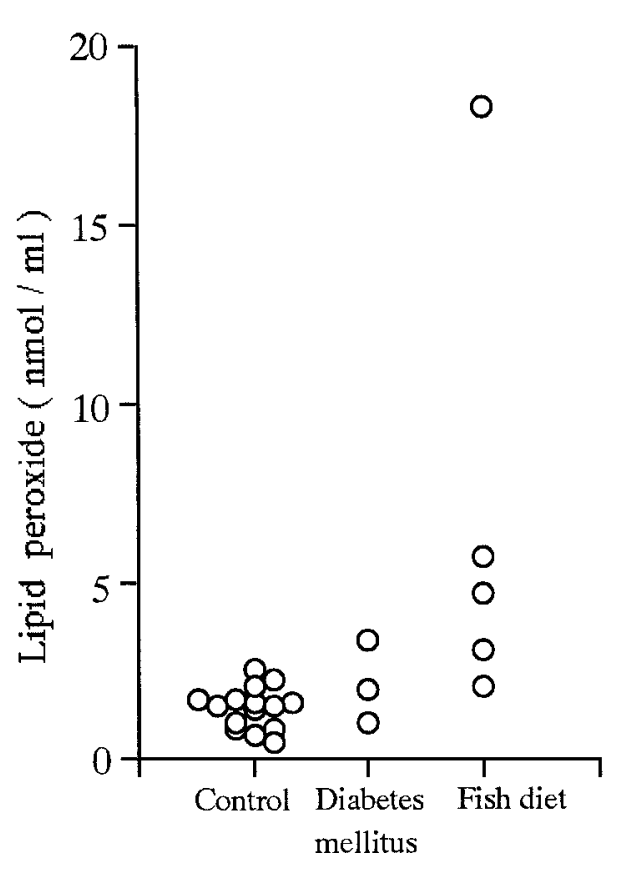

Fig. 1. Plasma lipid peroxide levels in normal cats $(n=15)$, cats with diabetes mellitus $(n=3)$, and cats fed a raw fish diet $(n=5)$.

upper limit of normal subjects $(3.0 \mathrm{nmol} / \mathrm{m} l)$. Four of the five cats fed a raw fish diet had a higher plasma lipid peroxide level $(3.1,4.7,5.7$ and $18.4 \mathrm{nmol} / \mathrm{ml})$ than the upper limit of normal subjects. The cats with the highest lipid peroxide value $(18.4 \mathrm{nmol} / \mathrm{m} l)$ were diagnosed as having pansteatitis, another two with chronic renal failure and one was infected with FIV.

Plasma lipid peroxide levels in cats fed a raw fish diet: Since a raw fish diet raised the plasma lipid peroxide levels in cats, we examined the effect of the diet in controlled conditions. Five clinically healthy cats which had been previously fed commercial canned food, were switched to a raw fish diet and the plasma lipid peroxide level was evaluated by the TBA method. As shown in Fig. 2, the plasma lipid peroxide level increased within 1 to 3 weeks to 1.9 to 15.3 $\mathrm{nmol} / \mathrm{ml}$. The plasma lipid peroxide level exceeded the upper limit of normal subjects in four of five cases. Then the level of plasma lipid peroxide was restored to the normal level even with the continuous raw fish diet (Fig. 2). Similar results were obtained in another experiment in which another five cats were fed a raw fish diet and plasma lipid peroxide levels were determined by the MCDP-Hb method (data not shown). To investigate whether the increased lipid peroxide was produced in vivo, or in vitro, we fed raw mackerel compulsively (approximately, $60 \mathrm{~g} / \mathrm{kg}$ ) and evaluated the plasma lipid peroxide levels continuously. Three cats were used and experiments were performed for each cat: before and after changing their diet to raw fish. These three cats had been fed normal canned food and then changed to a

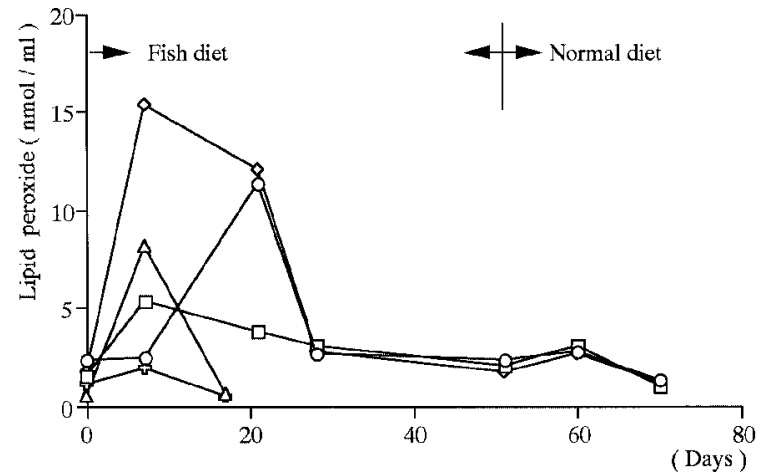

Fig. 2. Raw fish diet induced an increase in plasma lipid peroxide. Five clinically healthy cats were put on a raw fish diet for 1-6 weeks.

raw fish diet for 17 to 30 days. Plasma lipid peroxide levels were measured by the MCDP-Hb method (reference range for 10 control cats: 0.5 to $1.3 \mathrm{nmol} / \mathrm{ml}$ ). Plasma lipid peroxide levels in the cats did not exceed the upper limit of the normal range $(1.3 \mathrm{nmol} / \mathrm{m} l)$, before changing their diet to raw fish, but it exceeded the upper limit $(1.9,5.6 \mathrm{nmol} / \mathrm{ml})$ in two of three cats after changing the diet (Fig. 3). This increase was transient and was reduced within hours.

Peroxidation of plasma lipids in cats fed a raw fish diet: To estimate the potential plasma lipid peroxide, blood samples were reacted with air by gentle and continuous stirring for $5 \mathrm{hr}$ at $38^{\circ} \mathrm{C}$. Plasma samples were obtained after the reaction and the lipid peroxide levels were evaluated by the MCDP-Hb method. We used 3 cats which were fed a normal canned food and 4 cats fed a raw fish diet for 7 to 30 days. Potential lipid peroxide levels in the cats fed normal food remain relatively low, below $2.0 \mathrm{nmol} / \mathrm{ml}$, whereas those in cats fed a raw fish diet were increased remarkably, up to 2.3 to $30.4 \mathrm{nmol} / \mathrm{ml}$ (Fig.4).

\section{DISCUSSION}

Plasma lipid peroxide levels in clinical healthy cats were the same as those in humans. It was reported that plasma lipid peroxide levels in human patients with diabetes mellitus were higher than those in normal subjects $[5,7]$. In two of three cats with diabetes mellitus examined, the plasma lipid peroxide levels did not exceed the upper limit of the normal range. On the other hand, plasma lipid peroxide levels in field cases fed a raw fish diet as the principal food, were higher than the upper limit of normal in three of the four cats. One cat with pansteatitis had a remarkably high plasma lipid peroxide value. These results indicated that an increase in the plasma lipid peroxide level in cats could indicate abnormal lipid peroxide provoked by a diet. To confirm these results, we put some cats on a diet of raw fish. We have thought that vitamin E deficiency, which should develop months after beginning the diet, might be related to the increase. Plasma peroxide levels unexpectedly 

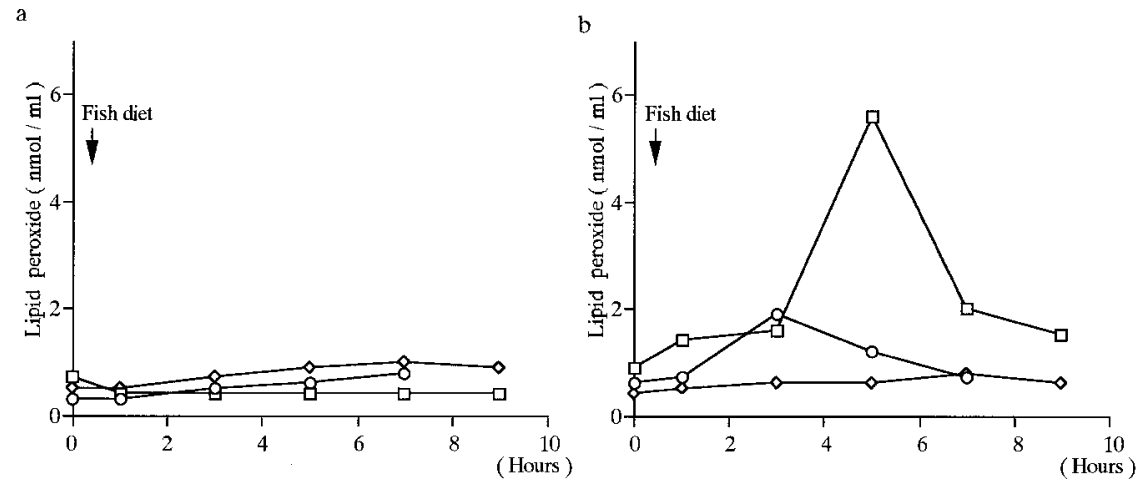

Fig. 3. Change in plasma lipid peroxide levels after being fed a raw fish diet. a, Plasma lipid peroxide levels in three cats which had been fed commercial canned food high in meat content. b, Plasma lipid peroxide levels in three cats which had been fed raw fish for 17-30 days.
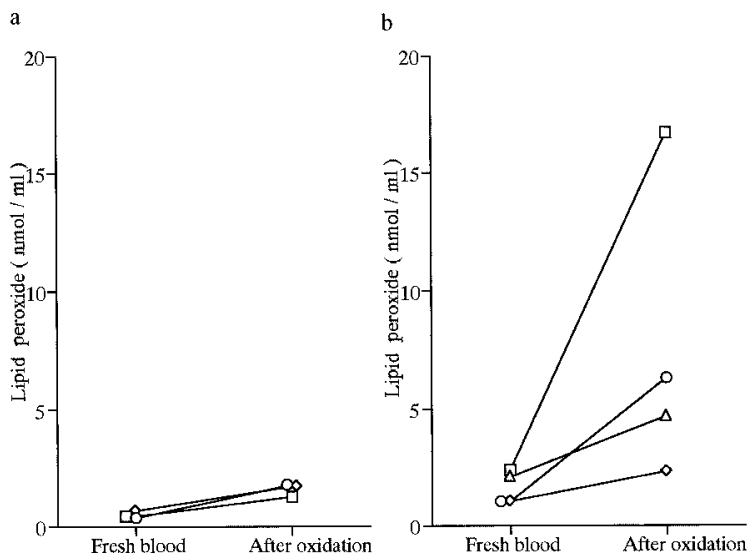

Fig. 4. Lipid peroxide generated by oxidation of blood samples. Blood samples were rotated and reacted with air for $5 \mathrm{hr}$ and plasma lipid peroxide levels were evaluated. a, Blood samples were collected from three cats which had been fed commercial canned food high in meat content. b, Blood samples were collected from four cats which had been fed a raw fish diet.

increased rapidly within 1 to 3 weeks in 4 of 5 cats. We also measured plasma vitamin E ( $\alpha$-tocopherol) levels in three cats and they did not change significantly during the experiment (plasma vitamin E levels before and after changing the diet were $12,13,13 \mu \mathrm{g} / \mathrm{m} l$ and $13,12,11 \mu \mathrm{g} / \mathrm{m} l$, respectively). We also observed that the increase occurs even when $\alpha$-tocopherol was added to a raw fish diet in a preliminary study. We therefore concluded that the transient increase in lipid peroxide is not related to vitamin E deficiency. We intended to clarify whether the increased lipid peroxide was generated exogenously, or produced in vivo by some peroxidative process in the cats. Plasma lipid peroxide levels of two of the three cats which had been fed raw fish increased within hours after feeding a raw fish diet, whereas those of the cats which had been fed normal cat food, did not increase. This result indicated that the rapid increase in lipid peroxide was not caused merely by the absorption of exogenously formed lipid peroxide. We also showed that potential lipid peroxide, probably polyunsaturated fatty acid, accumulated in cats fed a raw fish diet. We therefore supposed that small amounts of lipid peroxide formed exogenously would be absorbed and begin a radical chain reaction in the body of the cats in which polyunsaturated fatty acid accumulated. The increased lipid peroxide in the blood might attack the blood vessels and intact organs and tissues to induce various diseases such as vascular disorder or liver diseases. An extremely high lipid peroxide level is important because it causes secondary disorders by itself. It is also worth investigating whether the same phenomenon occur in another species, for example, humans. Recently, dietary supplementation of polyunsaturated fatty acid has been used for companion animals in a therapeutic way, for renal disease or dermatoses [1, 8, 9, 13]. Special attention must be paid to whether the dietary supplementation of polyunsaturated fatty acid causes oxidative stress.

It is interesting that the high plasma lipid peroxide level decreased even on a continuous raw fish diet. These results indicated that some antioxidant processes might develop in these cats. The increased lipid peroxides in the blood cannot be excreted into the urine, but are decomposed by enzyme systems such as the glutathione peroxidase-glutathione reductase system [19]. We examined the activity of plasma superoxide dismutase and glutathione peroxidase, but they did not change significantly during the period (data not shown). It would be beneficial to determine the mechanism by which increased lipid peroxides in the blood are eliminated because it may introduce a new therapeutic strategy for animal and human diseases related to lipid peroxides and free radicals.

In conclusion, the intake of food high in polyunsaturated fatty acid dramatically increases plasma lipid peroxide in cats. This is the first report, to our knowledge, in which the diet rapidly increased the plasma lipid peroxide level. In this study, no cat fed a raw fish diet showed signs of any clinical abnormalities during the period, but more attention should be paid to the effect of the diet containing polyunsat- 
urated fatty acid on generalized oxidative stress. Some cat diseases such as retinal atrophy and hepatic lipidosis may be caused by lipid peroxidation due to the diet. We should also investigate the potential pathogenicity of lipid peroxide and its relation to cat diseases.

ACKNOWLEDGMENT. This study was supported in part by Japan Forum on Small Animal Clinical Nutrition.

\section{REFERENCES}

1. Brown, S.A., Finco, D.R. and Brown, C.A. 1998. Is there a role for dietary polyunsaturated fatty acid supplementation in canine renal disease? J. Nutr. 128 (12 Suppl.): 2765-2767.

2. Demitsu, T., Katayama, H. and Yaoita, H. 1988. Serum lipid peroxide levels in various dermatoses. J. Dermatol. 15: 325329.

3. Di Luzio, N.R. and Hatman, A.D. 1967. Role of lipid peroxidation in the pathogenesis of the ethanol-induced fatty liver. Fed. Proc. 26: 1436-1442.

4. Fukuzumi, K. 1986. Relationship between lipoperoxides and diseases. J. Environ. Pathol. Toxicol. 6: 25-56.

5. Hayakawa, M. and Kuzuya, M. 1990. Free radicals and diabetes mellitus. Nippon Ronen Igakkai Zasshi 27: 149-154 ( in Japanese ).

6. Henning, B. and Chow, C.K. 1988. Lipid peroxidation and endotherial cell injury: implication in atheroscleroses. Free Radic. Biol. Med. 4: 99-106.

7. Kato, K., Midorikawa, S. and Watanabe, T. 1998. Evaluation of lipid peroxides in diabetes mellitus. Nippon Rinsyo 56: 121-125 (in Japanese).

8. Logas, D., Beale, K.M. and Bauer, J.E. 1991. Potential clinical benefits of dietary supplementation with marine-life oil. $J$.
Am. Vet. Med. Assoc. 199: 1631-1636.

9. Miller, W.H. 1989. Fatty acid supplements as anti-inflammatory agents. pp. 563-565. In: Current Veterinary Therapy X (Kirk, R.W. ed.), W. B. Saunders, Philadelphia.

10. Ohishi, N., Ohkawa, H., Miike, A., Tatano, Y. and Yagi, K. 1985. A new assay method for lipid peroxides using a methylene blue derivative. Biochem. Int. 10: 205-211.

11. Poli, G., Albano, E.and Dianzani, M.U. 1987. The role of lipid peroxidation in liver damage. Chem. Phys. Lipids 45: $117-142$.

12. Rikans, L.E. and Hornbrook, K.R. 1997. Lipid peroxidation, antioxidant protection and aging. Biochemi. Biophys. Acta 1362: 116-127.

13. Scott, D.W., Miller, W.H. Jr., Reinhart, G.A., Mohammed, H.O. and Baglade, M.S. 1997. Effect on an omega-3 / omega6 fatty acid- containing commercial lamb and rice diet on pruritus in atopic dogs: results of a single-blinded study. Can. J. Vet. Res. 61: 145-153.

14. Taki, M., Majima, A. and Takada, M. 1983. Experimental studies on retinopathy of prematurity. The morphological distribution of lipid peroxide in the kitten retina after oxygen administration. Nippon Ganka Gakkai Zasshi 86: 1245-1250 ( in Japanese).

15. Tapel, A.L. 1973. Lipid peroxidation damage to cell components. Fed. Prac. 32: 1870-1874.

16. Ungemach, F.R. 1987. Pathobiochemical mechanisms of hepatocellar damage following lipid peroxidation. Chem. Phys. Lipid 45: 171-205.

17. Yagi, K. 1976. A simple fluorometric assay for lipoperoxide in blood plasma. Biochem. Med. 15: 212-216.

18. Yagi, K. 1987. Lipid peroxides and human diseases. Chem. Phy. Lipids 45: 337-351.

19. Yagi, K. 1994. Lipid peroxides and related radicals in clinical medicine. Adv. Exp. Med. Biol. 366: 1-15. 